\title{
AGENTES COMPUTACIONALES Y ANÁLISIS ECONÓMICO
}

Juan Manuel Larrosa*

H $\mathrm{n}$ ciencias sociales, incluida la economía, hoy se pueden crear Dundos artificiales estableciendo un marco específico y creando entidades programadas que siguen ciertas reglas para responder preguntas hipotéticas analizando sus interacciones.

La simulación tradicional proyecta y manipula formas estilizadas agregadas para representar hechos sociales. Pero los fenómenos macro -las multitudes, el tráfico y el mercado de valores- surgen de la interacción entre agentes individuales. $\mathrm{El}$ agente intenta cumplir sus objetivos; puede ser una bacteria, una planta, familias, empresas o naciones. En la simulación se aplica cierta función o regla a un conjunto de entradas para obtener resultados ${ }^{1}$. Una manera de simular sistemas socio-económicos es la modelación basada en agentes múltiples, o multiagente, que interactúan en un ambiente dado, siguiendo ciertas reglas de conducta ${ }^{2}$. En los modelos basados en agentes (MBA) estos se formalizan como entidades que toman decisiones según sus objetivos, la información sobre el entorno y sus expectativas sobre el futuro. Los objetivos, la información y las expectativas son afectados por la interacción con otros agentes ${ }^{4}$, que pueden ser adaptativos, es

* Doctor en Economía, Departamento de Economía, Universidad Nacional del Sur, Bahía Blanca, Argentina, Instituto de Investigaciones Económicas y Sociales del Sur, [jlarrosa@criba.edu.ar]. Fecha de recepción: 9 de julio de 2014, fecha de modificación: 2 de marzo de 2015, fecha de aceptación: 12 de mayo de 2016. Sugerencia de citación: Larrosa, J. M. "Agentes computacionales y análisis económico", Revista de Economía Institucional 18, 34, 2016, pp. 87-113. DOI: http:// dx.doi.org/10.18601/01245996.v18n34.07

${ }^{1}$ Drogoul et al. (2003) revisan diversos enfoques para hacer simulaciones.

${ }^{2}$ El libro de Wooldridge (2009) es una buena introducción técnica a los sistemas multiagente (SMA).

${ }^{3}$ E1 término MBA aquí se usa para referirse a la modelación basada en agentes o a modelos basados en agentes.

${ }^{4}$ Esas interacciones endógenas se resumen en Vriend (2006). 
decir, que pueden cambiar sus decisiones y su comportamiento; o cognitivos. Los SMA tienen en cuenta características de los mercados reales como la heterogeneidad de los agentes, su racionalidad limitada y sus complejas interacciones; y dan una gran flexibilidad para modelar los fenómenos del mundo real. Quizá hagan posible una modelación más realista de los mercados y de la economía en su conjunto.

Este trabajo revisa algunos aspectos teóricos y prácticos de la MBA y los SMA aplicados a la economía; un campo aún poco explorado en la literatura en castellano, pero muy prometedor. Quizá esta revisión despierte el interés del lector para que incursione en esos y otros campos asociados que aquí no se consideran. En la primera sección se exponen los conceptos básicos. La segunda, de carácter descriptivo más que analítico, revisa algunas aplicaciones en macroeconomía y microeconomía. La tercera discute sus ventajas y sus dificultades, y la última presenta las conclusiones.

\section{QUÉ SON LOS AGENTES COMPUTACIONALES}

Los SMA y la MBA de sistemas socio-económicos aprovechan los avances del hardware -velocidad creciente de los procesadores y aumento de la capacidad de almacenamiento- y del software, como la programación orientada a objetos (POO), que hoy permiten modelar millones de interacciones simultáneas. Multitud de resultados de pequeña escala se han ampliado a escalas bastante mayores.

Un ejemplo clásico es el modelo de segregación de Schelling (1978), quien lo elaboró para entender la formación de patrones complejos de segregación urbana a partir de reglas simples de comportamiento. En una grilla ubicó individuos de dos tipos que desean vivir rodeados por vecinos de tipo similar (en algún porcentaje). Cuando la mayoría de los vecinos era del otro tipo, el agente se podía mudar iterativamente por toda la grilla hasta satisfacer sus preferencias. Aunque el experimento original era poco sofisticado y se hizo en una cuadrícula de papel con monedas de uno y diez centavos, que se desplazaban siguiendo esos criterios, los patrones de segregación surgían rápidamente. Con la posibilidad de programar sistemas de agentes, los resultados se replicaron a escalas que jamás imaginó su autor, y la mayoría de sus conclusiones se mantuvo (Vinkovic y Kirman, 2006). La gráfica 1 muestra una versión simple hecha por computador.

Como ya se dijo, técnicamente los SMA se derivaron de una rama de la informática que aprovechó los avances de la POO para modificar el enfoque de la simulación (Gilbert, 2008). Si bien no hay consenso en la definición de "agente", el término se suele usar para describir 
Gráfica 1

Modelo de segregación de Schelling

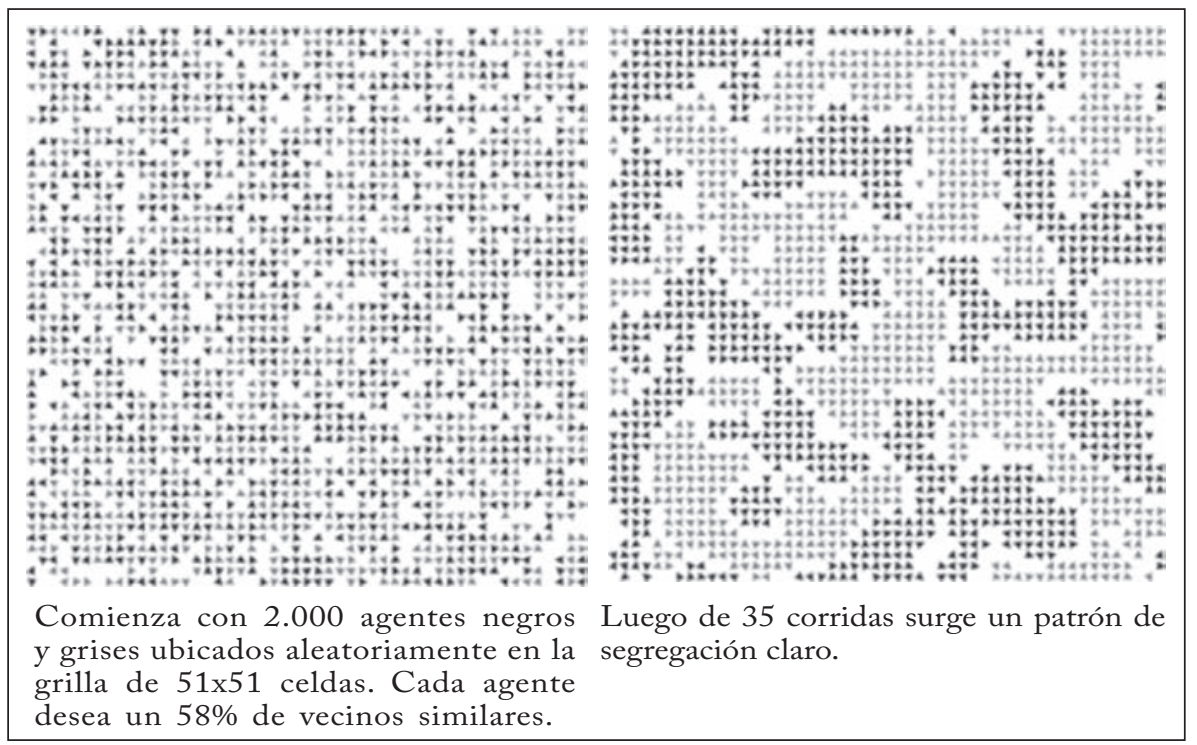

Fuente: elaboración del autor usando Netlogo.

los programas que controlan sus propias acciones basándose en su percepción del entorno operativo (Huhns y Singh, 1998). El objetivo es crear programas que interactúen "inteligentemente" con su entorno. En el diseño de estos agentes han influido los avances en inteligencia artificial (IA), sobre todo en la inteligencia artificial distribuida (IAD). El cuadro 1 muestra las propiedades asociadas a la definición débil de agente, tal como las sintetizan Russell y Norvig (2004).

Cuadro 1

Propiedades asociadas a la definición débil de agente

\begin{tabular}{ll}
\hline Propiedad & Descripción \\
\hline Autonomía & $\begin{array}{l}\text { La capacidad para controlar sus propias accio- } \\
\text { nes es asincrónica. }\end{array}$ \\
Reactividad & $\begin{array}{l}\text { Responde a cambios en el ambiente y decide } \\
\text { cuándo actúa. }\end{array}$ \\
Proactividad & $\begin{array}{l}\text { Responde del mejor modo posible a las posibles } \\
\text { acciones futuras que anticipa. }\end{array}$ \\
Habilidad social (habilidad Puede comunicarse en forma compleja con \\
para comunicarse) \\
otros agentes, incluidas personas reales, para \\
obtener información y lograr sus objetivos. \\
Habilidad para fijar objetivos Tiene un propósito. \\
Continuidad temporal & El agente es un proceso continuo en ejecución.
\end{tabular}

En suma, un agente es autónomo, reactivo, activo, comunicativo, automotivado y temporalmente continuo. Una vez diseñado y acti- 
vado, se debe observar y medir su comportamiento. Así, los MBA son representaciones abstractas de la realidad, en los que los objetos: a) interactúan entre sí y con el medio ambiente; b) son autónomos, es decir, no hay un control central de su comportamiento, y c) el resultado de su interacción se evalúa numéricamente. Por ser autónomos se los llama "agentes".

Este método no está exento de críticas en economía. Axtell (2000) y Richiardi (2004) resumen la discusión sobre los problemas básicos de diversos métodos: robustez, identificación, equifinalidad, ajuste excesivo, etc. La simulación con agentes es hoy una manera de hacer ciencia y comparte el devenir de los demás métodos. Primero se postula un modelo (es decir, se establecen las condiciones iniciales) y después "se resuelve". Una de las fortalezas de los SMA es que el sistema en su conjunto no está obligado a seguir un comportamiento particular pues este surge de la interacción entre los agentes. Por ello no se necesita suponer linealidad, equilibrio, transición suave, etc. E1 modelo se resuelve corriéndolo y se valida comparando sus resultados conforme al propósito de la investigación.

Según Axtell (2000), en ciencias sociales los agentes computacionales pueden tener tres usos: 1) computación numérica de modelos analíticos, es decir, introducción de valores en un modelo analítico para observar su comportamiento dentro del rango de valores admisibles;2) comprobación de la robustez de modelos analíticos, es decir, determinar para qué valores son válidas las conclusiones del modelo analítico y para qué valores no son válidas. Estos usos complementan el análisis matemático; el uso 3) es la simulación pura, es decir, modelar problemas analíticamente no formalizables o para los cuales no es útil la solución analítica. En este caso, la simulación sustituye al análisis matemático y es aquí donde surgen conclusiones originales. Para elaborar un modelo de simulación pura se requiere información específica sobre los agentes que se diseñan y de los comportamientos que se modelan.

Otra manera de describir un SMA es representarlo como una red de agentes en interacción, cuyas propiedades surgen por la interacción entre los agentes locales, generalmente heterogéneos, que forman su propio entorno. Hay modos aparentemente similares de estudiar este tipo de sociedades artificiales, por ejemplo, usando modelos de autómatas celulares (AC), donde los agentes se sitúan en una grilla, cada agente/célula contiene un decisor único, idéntico e inmóvil, y las reglas para tomar decisiones son idénticas ${ }^{5}$. Cada agente/célula es

\footnotetext{
${ }^{5}$ Heymann et al. (2013) y Gilbert y Troitzsch (2005) dedican sendos capítulos a $\operatorname{los}$ AC.
} 
potencialmente afectado por una externalidad espacial generada por las células inmediatamente vecinas. Aquí los agentes siguen siendo homogéneos durante la simulación. La homogeneidad ex post y ex ante es la principal diferencia entre la simulación con SMA y AC. Sin embargo, cabe observar que inmovilizando a cada agente el investigador puede restringir o circunscribir la externalidad que provocan sus vecinos para identificarla y medirla con más precisión.

E1 investigador de SMA empieza construyendo una economía con una población inicial de agentes (Tesfatsion, 2001), que pueden ser agentes económicos (comerciantes, entidades financieras, etc.) y agentes que representan factores sociales y ambientales (tierra, clima, etc.). Luego especifica el estado inicial de la economía dando valores a los atributos de los agentes. Estos pueden incluir normas de conducta internalizadas, modos de comportamiento internos (p. ej., de comunicación y aprendizaje), información almacenada acerca de sí mismo y de otros agentes (gráfica 2). Helbing (2012) hace una lista de posibles atributos que incluye: nacimiento, muerte y reproducción, necesidad de recursos (p. ej., para comer y beber), capacidad de competir, luchar y producir, grado de percepción del entorno, expectativas, capacidad de movilizarse y de comunicarse, entre otros. Una vez diseñado el agente, su versatilidad solo depende de las necesidades del investigador. Y una vez "nace" el agente computacional tiene una "vida virtual": enfrenta restricciones, desarrolla relaciones y opera con información limitada, parecida a la vida de un agente real. Todos los eventos que luego ocurran emergen siguiendo la línea temporal de las interacciones entre agentes. En los MBA no se permiten mecanismos de coordinación exógenos, pues las regularidades globales surgen a partir de los microfundamentos mediante la repetida interacción local de agentes autónomos.

A veces el objetivo es reproducir comportamientos agregados (complejos) a partir de agentes autónomos con racionalidad limitada. Otras veces el propósito es más ambicioso: diseccionar fenómenos emergentes mediante ingeniería inversa para identificar los patrones de conducta individual que generan sistemas tales como colonias de hormigas, un sistema económico particular o el sistema inmunológico, usando herramientas de teoría de juegos, de la economía o la biología. Las ideas y algoritmos de la inteligencia artificial se complementan con investigación sobre planificación, métodos de razonamiento, de búsqueda y aprendizaje automático, para completar el diseño de la conducta de los agentes. 
Gráfica 2

Esquema de un modelo SMA

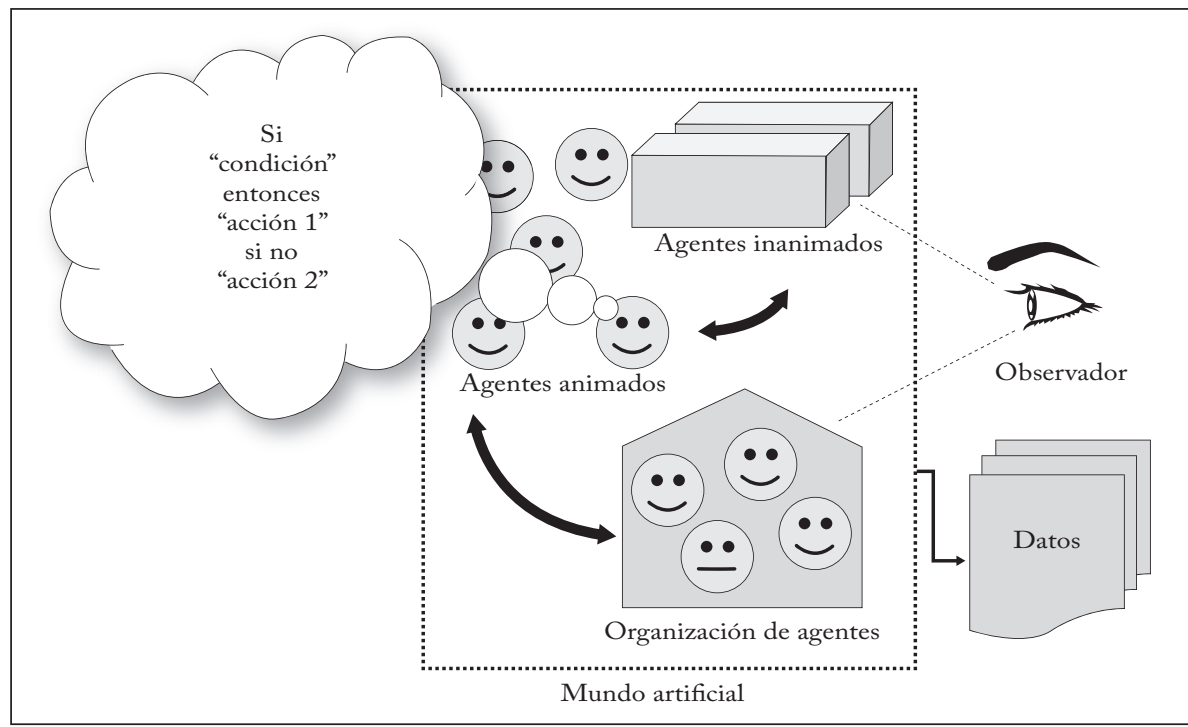

Fuente: Swarm tutorial.

El investigador debe identificar inicialmente los aspectos esenciales del diseño del agente y de su entorno. En un SMA algunas características del entorno podrían incluir:

- Conocimiento y creencias: los agentes basan sus acciones en lo que saben de su entorno (incluidos los agentes de otro tipo).

- Inferencia: dado un conjunto de creencias, los agentes pueden inferir más información. Por ejemplo, si el agente B acaba de "comer" algunos "alimentos", el agente A puede inferir que el lugar para encontrarlos está cerca de donde se localiza B.

- Modelos sociales: algunos agentes pueden aprender acerca de la interrelación entre otros agentes; por ejemplo, de la interacción entre los agentes A y B.

- Representación del conocimiento: para construir sus modelos, el agente necesita representar sus creencias. Las técnicas para ello han sido estudiadas en inteligencia artificial, bajo el epígrafe de "representación del conocimiento".

- Objetivos: como los agentes son intencionados y autónomos, para realizar una acción deben ser inducidos por la necesidad de satisfacer una meta interna, como la supervivencia. La que a su vez requiere satisfacer objetivos subsidiarios, como adquirir energía y evitar peligros letales. 
La modelación tiene varias etapas que transcurren a medida que el bosquejo inicial se ejecuta, se analiza y se corrige iterativamente (Izquierdo et al., 2008). El modelador diseña un primer modelo que arroja unos resultados iniciales. A la luz de esos resultados, vuelve a las etapas iniciales para perfeccionarlo revisando algunas premisas. Es posible que considere insatisfactorios los resultados iniciales: a) porque no parecen derivarse lógicamente de las premisas (verificación) o porque -aun siendo lógicamente correctos- difieren demasiado de las observaciones del sistema real (validación) ${ }^{6}$. Revisemos brevemente estos dos procesos.

- Verificación: consiste en comprobar que el modelo cumple los requisitos de diseño impuestos por el modelador (Kleijnen, 1995; Sargent, 2010). En forma intuitiva, verificar un modelo es asegurar que es lo que su diseñador piensa que es. La verificación es esencial en la elaboración de modelos formales, en los que la implementación (en un lenguaje formal) y la derivación lógica de resultados (mediante deducción) no están exentas de errores. La primera implementación de un modelo computacional suele producir resultados incoherentes con las premisas, por ejemplo, que una variable tome valores fuera del rango admisible (como un porcentaje negativo). Esto indica que ha habido un error de programación y, por tanto, que el código se debe revisar. Esta revisión, que no hace referencia alguna al mundo real, es parte del proceso de verificación.

- Validación: a diferencia de la verificación, la validación hace referencia explícita al sistema real que se está modelando. Validar un modelo consiste en evaluar su utilidad en el contexto de aplicación, según los criterios para los que fue diseñado. En el ámbito científico, el propósito de construir modelos (y el criterio para evaluarlos) suele ser mejorar el conocimiento de un sistema real dado. Así, si el objetivo es representar de modo fiel un proceso social, el modelo será tanto más válido cuanto mejor capte la esencia de su referente empírico. Lo anterior se puede evaluar examinando el grado en que los resultados del modelo se ajustan a los observados empíricamente (Moss et al., 1997).

De manera un tanto informal, podríamos decir que verificar consiste en valorar si el modelo que tenemos es correcto y validar consiste en estudiar si tenemos el modelo correcto (Izquierdo et al., 2008).

Los SMA/MBA abordan la heterogeneidad de los agentes en forma directa, y pueden modelar individualmente a cada agente. El sinónimo

${ }^{6}$ Ver Galán et al. (2009), quienes analizan las diferencias entre lo que el investigador piensa que hace el modelo y lo que hace verdaderamente. 
de la MBA es la modelación microscópica (Bonabeau, 2002), es decir, del detalle y la diferencia. Los investigadores pueden distinguir entre agentes, modelarlos conforme a cada tipo observado o a familias de agentes y hacer que interactúen. Lo que está en el centro de la MBA es el agente heterogéneo, no un agente representativo homogéneo. Según Borrill y Tesfatsion (2011), debido a esta ductilidad la MBA es la "matemática correcta" para el estudio de procesos en ciencias sociales.

En economía, por ejemplo. Las economías de mercado son subsistemas formados por un gran número de agentes adaptativos envueltos en interacciones locales simultáneas. Esa interacción genera regularidades microeconómicas y macroeconómicas -como las normas de comportamiento compartidas y los protocolos de mercado- que a su vez realimentan las interacciones locales. El resultado es una compleja dinámica sistémica de cadenas causales recurrentes que conectan comportamientos individuales, redes de interacción y resultados que afectan el bienestar social. En ese contexto se puede crear una diversidad de agentes capaces de tomar decisiones autónomas (aunque el número de acciones sea limitado) en interacción permanente. Por ejemplo, un agente "consumidor" cuyo comportamiento es un vector de disposiciones a pagar decrecientes por cantidades crecientes de un bien, y un agente "empresario" con un vector de costos marginales crecientes asociados a la producción de ese bien. Cada cual tiene información que solo él conoce y que utiliza para obtener la mayor utilidad posible. El consumidor intentará buscar el menor precio y el empresario, vender al mayor precio. En la interacción ambos agentes se contactan, con o sin costos de intercambio. Una vez en contacto, harán el intercambio si es mutuamente beneficioso.

Los investigadores de SMA y MBA recurren a laboratorios computacionales para estudiar la evolución de las economías de mercado en condiciones experimentales y explicar el comportamiento emergente (Gallego, 2007). ¿Por qué surgen regularidades globales y persistentes pese a la falta de control y de planificación central? ¿Cómo se generan estas regularidades globales, de lo micro a lo macro, mediante la interacción local repetida entre agentes autónomos? En la MBA y en los SMA y la interacción repetida genera patrones microeconómicos cambiantes que provocan cambios en la variable macroeconómica de interés. Muchos académicos auguran un futuro fructífero a la modelación basada en agentes (ver Farmer y Foley, 2009) $)^{7}$.

7 Para aplicaciones en diversas ciencias, ver Drogoul et al. (2003), Edmonds (2001) y Galán et al. (2009). Un buen compendio es Tesfatsion y Judd (2006). 


\section{SMA Y MBA EN ECONOMÍA}

Los aportes en este campo se pueden dividir según la clasificación clásica: de tipo agregado (macroeconómicos) y enfocados en individuos o un sector (microeconómicos). En los apartados siguientes se revisan algunos aportes importantes en esas áreas.

\section{SISTEMAS Y MODELOS AGREGADOS DE AGENTES EN ECONOMÍA}

Las ciencias sociales evolucionaron adoptando nuevos instrumentos y métodos para probar sus hipótesis teóricas y empíricas. En particular, el conocimiento económico ha avanzado adoptando nuevos enfoques para probar hipótesis sobre la asignación de recursos y la dinámica del mercado. Esto ha llevado al surgimiento de un cuerpo metodológico formal (representado en modelos matemáticos) que ha alimentado y dado forma a la teoría económica moderna. Por ejemplo, la teoría macroeconómica ha sacado conclusiones con base en modelos agregados en los que se adoptan supuestos simplificadores sobre el comportamiento de los agentes, representados por un agente representativo.

Se ha criticado esta manera de representar el mundo. La crítica de Lucas (1976) inició un embate metodológico contra los modelos muy agregados. Los críticos exigían que se basaran en supuestos microeconómicos (microfundamentos). El tratamiento simplista de las expectativas se corrigió a partir de esas críticas (Fagiolo et al., 2007). El análisis de casos donde surgen interacciones complejas muestra que la heterogeneidad, suprimida por un supuesto simplificador para obtener resultados estilizados, afecta los resultados del modelo (Kirman, 1992). La crisis de 2008 no solo mostró la importancia de los mercados financieros en el funcionamiento de la economía real. También demostró la insuficiencia de la teoría dominante: los modelos estándar no la predijeron. Y aún no sugieren medidas correctivas para restaurar el crecimiento (Stiglitz y Gallegati, 2011). Las limitaciones del individualismo metodológico se pueden superar usando SMA/MBA que no adoptan supuestos ideales sin sustento empírico. También son una alternativa a los modelos de agente representativo (Kirman, 1992) y establecen un vínculo natural entre la descripción micro y macro, considerando la heterogeneidad, la variabilidad y las fluctuaciones espacio-temporales, que modifican el comportamiento del sistema, a veces drásticamente.

Aunque los investigadores suelen utilizar SMA y MBA para modelar proposiciones derivadas de la teoría económica, a continuación 
se revisa un aporte más abstracto al estudio del comportamiento agregado: el de los mundos artificiales.

\section{Mundos artificiales}

En los trabajos sobre mundos artificiales se crean escenarios simples pero completos donde los agentes nacen, se desarrollan, interactúan y mueren, que permiten estudiar hechos estilizados. Epstein y Axtell (1996) es quizá uno de los más influyentes sobre la aplicación de MBA en ciencias sociales (gráfica 3).

Gráfica 3

Mundo artificial Sugarscape de Epstein y Axtell (versión NetLogo)
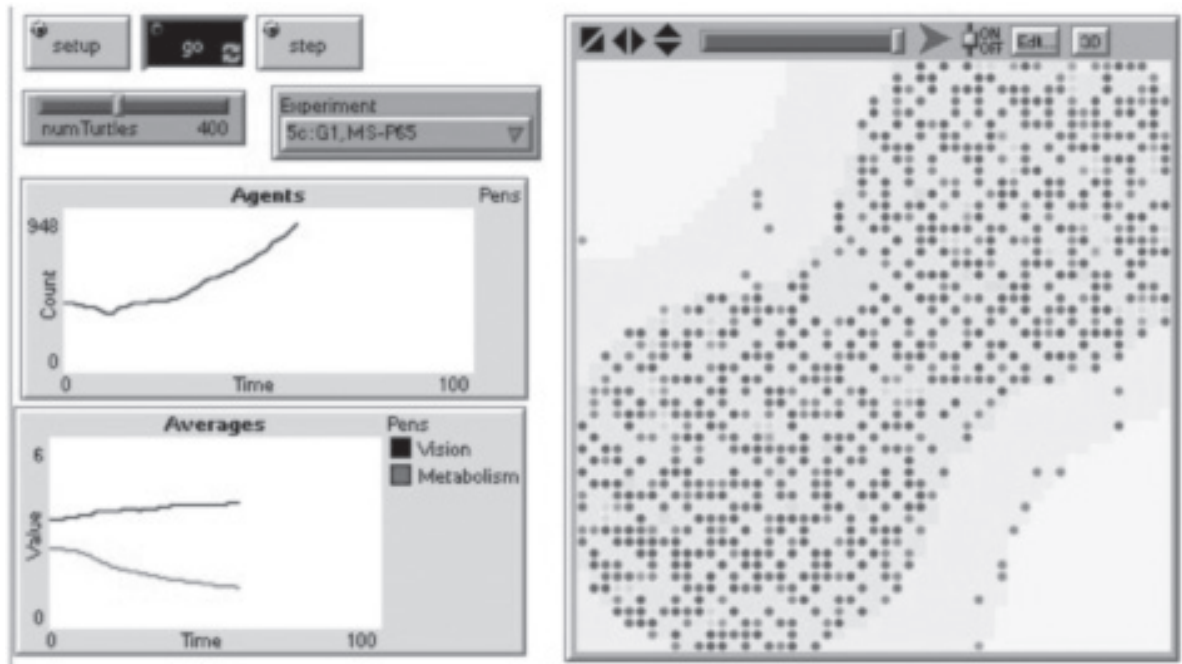

E1 modelo representa dos reservorios de especias, un bien que se puede usar como alimento, ubicados en las esquinas noreste y sudoeste. Al inicio los agentes se ubican aleatoriamente.

Fuente: biblioteca de modelos de Netlogo.

Estos autores crean un mundo con agentes en cuyos alrededores hay dos reservorios de alimento (sugar); en algunas configuraciones se produce un alimento de intercambio llamado especia (spice). Los agentes pueden comer, metabolizar, morir, generar prole y almacenarla si recolectan más de la que satisface sus necesidades. Ese excedente se puede usar para hacer intercambios. La interacción y el aprendizaje hacen posible que los agentes evolucionen e intercambien, se reproduzcan y desplacen, e incluso que surjan guerras. Este mundo artificial Sugarscape es uno de los experimentos in silico más influyentes y estimulantes para el investigador y sin duda condensa la categoría de mundo digital. 
Oremland y Laubenbacher (2014) emplean el Sugarscape como macroeconomía y hacen simulaciones para aplicar modelos de tributación óptima. La interacción entre ecuaciones en diferencias y MBA aprovecha lo que es mejor del análisis formal y de la simulación.

\section{Macroeconomía computacional basada en agentes}

La economía computacional basada en agentes (ECBA) es una de las ramas de mayor crecimiento en los últimos años (Tesfatsion y Judd, 2006). En un principio los modelos aplicados a estudios macroeconómicos eran escasos. Sin embargo, Bruun (2000) modeló un sistema macroeconómico con fundamentos keynesianos y obtuvo resultados agregados muy similares a los que predice la síntesis neoclásica. Paradójicamente, Bruun sigue la noción de macrofundamentos keynesianos más que la de microfundamentos. Primero, el modelo busca reproducir los resultados agregados esperados por la síntesis neoclásica y, luego, a partir de ellos, construir cada unidad individual para mejorar el ajuste. En este modelo, que se deriva de una modelación controlada, no hay agentes optimizadores. Metodológicamente parece similar al de Kydland y Prescott (1996), quienes elaboran un modelo (macro) y varían ciertos parámetros para que se ajuste a los datos de la realidad (calibración), y así obtienen los parámetros del agente representativo (microeconómico). Aparte de esta semejanza, el enfoque es totalmente diferente. Para Kydland y Prescott los agentes tienen información perfecta y los mercados son perfectamente competitivos. La interacción no genera fricciones ni externalidades; el resultado agregado es simplemente la suma de los resultados individuales. Para un MBA, ese es un caso muy particular y puntual, no el resultado general esperable.

El enfoque macroeconómico de Bruun se refina en la formulación de Bruun y Luna (2000), quienes encuentran fluctuaciones endógenas en un modelo basado en agentes de corte keynesiano. Este modelo tiene muchas semejanzas con el anterior, pero el diseño de cada agente es más complejo y le da un mayor margen de comportamientos posibles. Además, es un trabajo precursor en la traducción de las funciones de oferta y demanda, las decisiones de consumo y las condiciones de bancarrota a códigos programables. El resultado es una economía artificial con interacciones simultáneas que genera crecimiento y ciclos a partir de comportamientos microeconómicos e interacciones entre agentes claramente definidos. Bruun (2001) describe los efectos del crecimiento económico sobre la desigualdad de ingresos de los agentes. Un libro más reciente sobre la MBA aplicada al estudio 
de las fluctuaciones económicas es el de Gatti at al. (2008), donde se construye una economía agregada a partir de las empresas que la integran, y a cada paso se replican métricas reales (p. ej., la aparición de alta concentración en los sectores productivos).

Con la crisis del mercado hipotecario resurgió la crítica a la excesiva estilización en la modelización macroeconómica. Por ejemplo, Stiglitz y Gallegati (2011) argumentan que se debe enfrentar directamente la complejidad de las redes de interrelación en los mercados financieros y de crédito, en vez de eludir el problema usando supuestos simplificadores que no generan desequilibrios. Y proponen sustituir el agente representativo formal por agentes computacionales heterogéneos. Un compendio de estas aplicaciones al estudio de la macroeconomía moderna es la obra de Heymann et al. (2013), que trata muy bien los temas relacionados con SMA, en especial la generación de corridas bancarias y de acciones colectivas espontáneas.

\section{Modelos de interacción micro-macro en el mercado de trabajo}

Entre los estudios del mercado de trabajo que emplean modelos basados en agentes cabe mencionar los siguientes: Pingle y Tesfatsion (2003), Delre y Parisi (2007) y Tesfatsion (2001, 2002), así como el artículo de Fagiolo et al. (2004) que resume los aportes técnicos para explicar las regularidades observadas en los mercados de trabajo. En estos modelos se diseña algún tipo de interacción para generar las regularidades o hechos estilizados que se observan en los países desarrollados, como la curva de Beveridge (la relación negativa entre tasa de desempleo y tasa de oferta de empleo). Gabriele (2002) y Fagiolo et al. (2004) encuentran que esta curva solo aparece en una economía con emparejamiento (matching) cuando se simulan restricciones institucionales. Estas restricciones institucionales se enmarcan en escenarios donde las acciones futuras dependen débilmente de las acciones anteriores (dependencia débil de la trayectoria), por ejemplo, cuando las firmas requieren que el número de empleados dependa de los beneficios del último periodo y que los trabajadores busquen empleo, con cierta inercia, en la última firma que los empleó. Aunque estilizadas, estas restricciones al comportamiento de los agentes y a su interacción se pueden modificar para adaptarlas a otros escenarios. De este modelo se pueden sacar conclusiones sobre la evolución del producto bruto (ciclos y crecimiento). Por su parte, Leombruni y Richiardi (2005) elaboran un modelo del mercado laboral italiano para proyectar la evolución del porcentaje de jubilados dentro de la 
población económicamente activa. Y Neugart (2008) emplea la MBA para sugerir políticas alternativas contra el desempleo.

\section{MODELOS DE AGENTES EN MICROECONOMÍA}

Un aporte fundamental de la MBA a la teoría económica es sin duda el experimento de Gode y Sunder (1993), quienes examinan el comportamiento de agentes virtuales sin memoria, sin capacidad de maximizar ni de observar, recordar o aprender, a los que denominan agentes de inteligencia cero (IC). Estos actúan en una subasta virtual como compradores o vendedores emitiendo órdenes aleatorias. Un grupo tiene una restricción presupuestal (no vende a menos del costo ni compran a precios mayores de su propia valoración) y otro grupo no la tiene. Se incluye, además, un tercer grupo de tratantes humanos y se generan sesiones de subastas para observar la senda de equilibrio. El análisis muestra que el grupo de agentes de IC con restricción presupuestal obtiene un resultado de equilibrio semejante al del grupo de humanos. En suma, cuando los agentes computacionales actúan con la misma restricción que los tratantes humanos se logra un equilibrio, sin influencia de la memoria, la inteligencia o la motivación de lucro.

\section{Comportamiento del consumidor}

El comportamiento de los consumidores ha sido muy estudiado tanto en microeconomía como en análisis de mercadeo ${ }^{8}$. La MBA puede ayudar a encontrar respuestas a problemas aparentemente complejos ${ }^{9}$. Un ejemplo práctico es el mercado de ropa modelado por Brannon (2000), quien elaboró un MBA para representar la interacción entre factores externos (publicidad, promoción y colocación de productos) e influencia personal (comunicación directa), y entre líderes de la moda y seguidores de la moda. Los agentes son adaptativos; los efectos de los factores externos tienden a volverse difusos y dejan de ser importantes. Se fijan algunos parámetros y reglas de comportamiento para comparar los resultados con los de modelos de difusión de innovaciones.

Hay una bibliografía creciente sobre SMA y MBA aplicados a este campo. Baste mencionar dos trabajos sobre simulación de comportamiento del consumidor: Zhang y Zhang (2007) y Baptista et al. (2014), que elaboran modelos de agentes construidos con base en la teoría económica y el marketing.

\footnotetext{
${ }^{8}$ Ver una extensa revisión en Macal y North (2005).

${ }^{9}$ Ver, p. ej., Jager (2000), un trabajo muy citado por investigadores que modelan el comportamiento de los consumidores mediante MBA.
} 


\section{Supermercados}

Un estudio práctico es Venables y Bilge (1998), que adoptan el enfoque de SMA para entender el comportamiento de clientes reales creando agentes heterogéneos a partir de datos reales de un supermercado londinense. La información se recogió directamente del escáner del supermercado, al que tuvieron acceso, lo que permitió definir con precisión las categorías de clientes y las listas de compras con base en datos reales. Esa información les permitió incluso definir la edad del agente y el tiempo de compra en días precisos de la semana. Los agentes/clientes debían minimizar el tiempo que pasan en la tienda, completar la lista de compras, maximizar la compra de promociones, satisfacer sus restricciones de tiempo, considerar la posibilidad de sustituir bienes y evitar la congestión.

Los agentes siguen reglas simples ("dondequiera que estés, ve a la ubicación más cercana del primer elemento de la lista de compras”, "evita la congestión", etc.). Sobre un plano real del supermercado, los autores elaboraron un SMA llamado SimStore que genera los caminos que toman los clientes, a partir de los cuales se puede calcular la densidad de clientes en cada lugar para prever zonas de congestión, las ventas totales, la incidencia de las promociones, etc. Los agentes también se utilizan para probar propuestas de comercialización antes de ponerlas en práctica.

Siebers y Aickelin (2007), que aplican el enfoque de SMA a una cadena de supermercados centrándose en la relación entre gestión de recursos humanos y productividad, simulan dos políticas de personal (desarrollo de carrera y empoderamiento del personal) para prever los resultados en la operación de la firma. Schwaiger y Stahmer (2003) elaboraron un sistema más complejo, el SimMarket, en el que interactúan diversos agentes con niveles crecientes de complejidad, cuyo comportamiento es generado a partir de redes de inferencia bayesiana. Si bien es muy realista, las características de los agentes y las reglas de comportamiento de este modelo están muy lejos de ser tan simples como las del modelo de Schelling.

Por último, cabe mencionar la aplicación de SMA que desarrollaron Caramuta y Larrosa (2007) para replicar el desplazamiento de los carros de supermercado y entender la generación de atascos, el papel de la arquitectura del local y la disposición de los estantes. Con un SMA muy simple, simulan el ingreso de los agentes al supermercado con una lista de compras e intentan minimizar el recorrido entre los estantes donde se encuentran los bienes de la lista y las cajas registra- 
doras. Los resultados del modelo se comparan con los que se observan realmente para mejorar la gestión del supermercado.

\section{Mercado de estaciones de servicio}

Heppenstall et al. (2006) desarrollaron una interesante aplicación para modelar estaciones de gasolina (petrol stations en el Reino Unido) empleando un SMA. Aquí las estaciones de servicio individuales son agentes-objetos a los que se suministra información del precio inicial, los costos de producción y los precios de las estaciones cercanas. Los precios se toman de la realidad o se simulan, bien sea que el modelo se use para examinar comportamientos reales o abstractos. Cada agente observa los precios de las estaciones vecinas y aplica una serie de reglas para ajustar sus propios precios. La interrelación entre diferentes conjuntos de reglas lleva a que las estaciones compitan y actúen para maximizar los beneficios. Las estaciones actualizan sus precios sincrónicamente una vez al día y solo se interesan en su propio beneficio. Siguiendo reglas de conducta reactivas a las de las estaciones cercanas, fijan sus precios de acuerdo con la fluctuación de los beneficios. Si aumentan, mantienen el precio, y si bajan lo modifican, subiéndolo o bajándolo dependiendo de la elasticidad de demanda de cada tipo de cliente.

E1 otro tipo de agentes es el de los consumidores, para los que se elaboran tres modelos: un modelo MBA puro, uno de interacción espacial híbrido y uno de interacción espacial híbrido con red. Los cuales difieren en el grado de información que tiene el consumidor al comienzo. Los resultados indican que el modelo híbrido funciona un poco mejor que el primero, pues simula mejor la dinámica del sistema, aunque la representación del momento en que se carga el combustible es muy simplificada. Los mismos autores comentan que el combustible se suele comprar en camino hacia algún destino y la mayoría de las veces camino al trabajo.

\section{Cadena de suministros}

Las cadenas de suministros son particularmente interesantes para aplicar SMA. En su extensa revisión, Macal (2003) muestra cómo se diseña un modelo SMA de una cadena de suministro. Los agentes de la cadena son un cliente, un vendedor minorista, un vendedor mayorista, un distribuidor y un productor. En cada periodo, los agentes de la cadena siguen estas reglas simples: el cliente hace una orden al minorista; el minorista satisface la orden con su inventario si tiene 
suficiente (si se queda sin inventario, la pone en espera y la cumple cuando renueva el inventario); el minorista recibe un embarque del mayorista a pedido previo, decide cuánto ordenarle con base en una "regla de pedidos" y en su estimación de la demanda futura, para la cual usa una regla de "predicción de demanda". El minorista hace órdenes al mayorista para cubrir la demanda esperada y cualquier escasez bien sea por insuficiencias del inventario o los canales de distribución. De modo semejante, cada mayorista recibe un embarque del distribuidor, predice la demanda futura del minorista, y hace órdenes al distribuidor. En este ejemplo, los agentes de la cadena solo tienen acceso a información local. Ninguno tiene una visión global ni puede optimizar el sistema como un todo. Este modelo se ha usado para replicar la cadena de suministros del juego de simulación de la cervecería de Sterman (1989), que se realiza con personas reales.

Por su parte, Schenk et al. (2007) elaboraron un modelo de SMA de una verdulería regional en el que se utilizan redes sociales y variables espaciales. Los resultados de la simulación se validaron con una enorme base de datos de una empresa real. Joo et al. (2000) también modelaron una cadena de suministros on-line de verduras usando el enfoque SMA.

\section{Redes industriales y de intercambio}

La teoría económica suele pasar por alto la importancia de los vínculos entre empresas, pese a que las redes son de obvia importancia en la industria. Un ejemplo bien conocido son los “distritos industriales" en el norte de Italia, como Prato, el distrito de producción textil, donde un gran número de pequeñas empresas agrupadas en una pequeña región fabrica el mismo tipo de producto, con fuertes pero diferentes vínculos entre ellas: cliente/proveedor, de colaboración técnica, financieros o, simplemente, de amistad o relación familiar entre los dueños ${ }^{10}$.

Se puede crear un mercado virtual en el que muchos compradores y vendedores interactúan recurrentemente, aprenden unos de otros y toman en cuenta las interacciones previas. Ese mercado se puede usar para evaluar las proposiciones de la teoría económica neoclásica y elaborar nuevas teorías partiendo de sus mismos microfundamentos. Esta ha sido la tarea abordada por Tesfatsion $(1997,2002,2005)$, quien modela procesos de producción contemplando diversos aspectos micro, como la determinación de precios y el intercambio, la tasa de

\footnotetext{
10 Algunos trabajos que exploran esta aplicación de SMA y MBA son Albino et al. (2003), Boero et al. (2004), Borrelli et al. (2005), Brenner (2001), Fioretti (2004) y Squazzoni y Boero (2002).
} 
supervivencia de las firmas, la rivalidad estratégica y el poder de mercado, la incertidumbre del comportamiento y del aprendizaje, el papel de las convenciones y las organizaciones y las complejas interacciones entre los atributos estructurales del modelo.

Izquierdo et al. (2006) reproducen en algunos puntos un ejemplo más elaborado de una red de comercio para evaluar las fallas de mercado asociadas a la incertidumbre respecto de la calidad (gráfica 4). En el modelo hay dos tipos de agentes: compradores y vendedores; los compradores se relacionan a través de una red social (predeterminada). Cuando se observa la calidad del producto, la información viaja a través de la red social y afecta la percepción de los consumidores sobre la calidad de los bienes, la cual influye en su demanda en el mercado.

Gráfica 4

Red de intercambio con incertidumbre respecto de la calidad

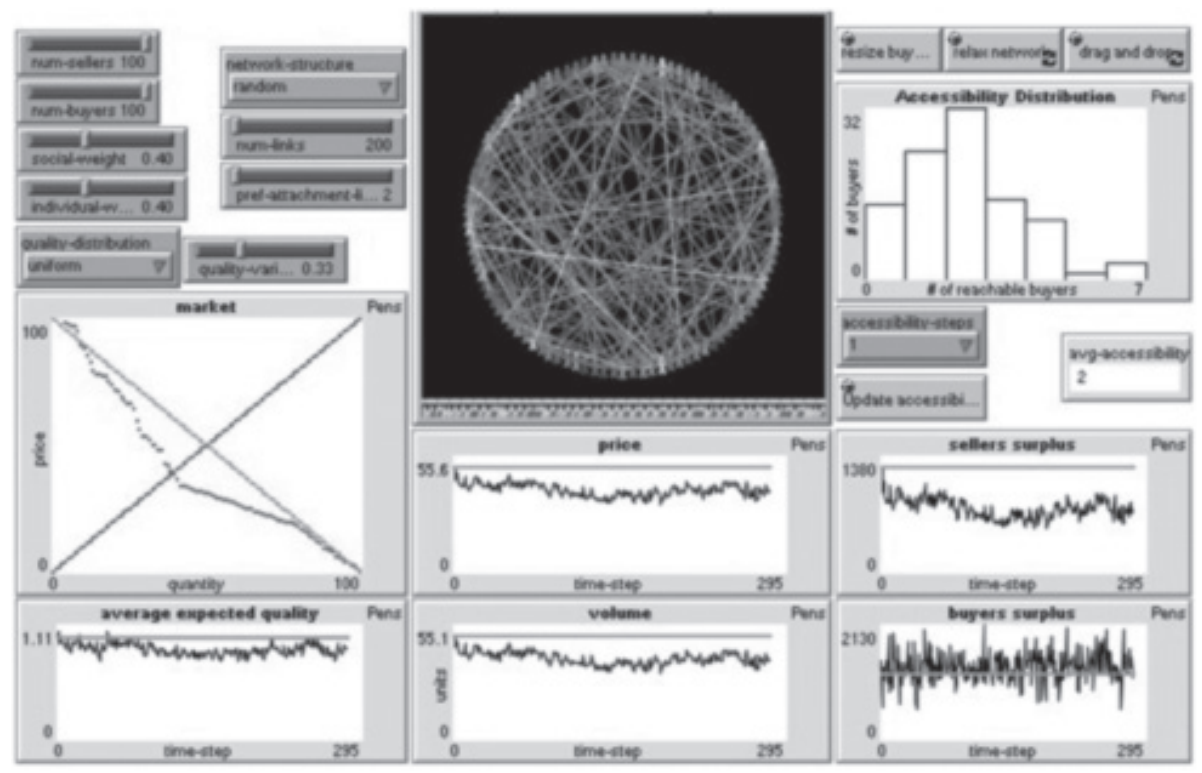

El modelo se inicia cuando los compradores forman una red social elegida por el observador (arquitectura de círculo, aleatoria, enlace preferencial o estrella). Los vendedores ofrecen su producto de acuerdo con el costo marginal. Los compradores compran según su disposición a pagar, que es afectada por la calidad del producto, percibida y observada. Estas se transmiten a los demás compradores a través de la red social.

Fuente: código Netlogo generado por Izquierdo et al. (2006).

Gatti et al. (2008) modelan fluctuaciones económicas, y su obra se fundamenta en la simulación de la dinámica industrial de diversos sectores productivos. Su propósito es explicar las fluctuaciones debidas 
a choques sectoriales y para ello elaboran un modelo MBA que genera fluctuaciones agregadas.

Otro ejemplo de modelación de industrias en red, específicamente de redes eléctricas, con MBA es Li et al. (2010). Un esfuerzo enorme de recreación del sistema eléctrico estadounidense, representando mediante agentes a cada nodo de oferta y demanda de electricidad. La simulación detecta puntos de ineficiencia en el uso de la capacidad instalada de la red (de congestión y de precios mayores que el costo marginal de suministro), así como situaciones de poder de mercado de los proveedores mayoristas en distintos puntos de la grilla eléctrica.

\section{Telecomunicaciones y difusión de tecnologías}

Collings et al. (1999) usan MBA para estudiar el ritmo de adopción de servicios de telecomunicación. Los modelos tradicionales de difusión no pueden describir la forma en que los atributos de los miembros del sistema social dan lugar a la tasa observada, a la extensión o a la magnitud de la difusión. Un tema que estos autores examinan expresamente es cómo interactúan los clientes para aprender acerca de un servicio a través de sus comunicaciones mutuas. Utilizando características del agente obtenidas de la base de datos de interacciones reales muestran que la estructura, la dinámica y la distribución de las redes sociales influyen en la difusión del servicio. El modelo indica que las tasas de adopción de un servicio de telecomunicaciones en el mundo real dependen de esas características de la red, que interactúan en forma no lineal. Los autores argumentan que el enfoque de sistemas complejos es muy útil para descomponer esas interacciones. Twomey y Cadman (2002) revisan diversos trabajos sobre los mercados de CD, telecomunicaciones, subscripción a TV paga, etc. Otro trabajo digno de mención es el de Pegoretti et al. (2012), quienes modelan procesos de difusión de innovaciones mediante agentes que interactúan en redes sociales con estructura de mundo pequeño.

\section{DIFICULTADES Y DEBILIDADES DEL USO DE SMA/MBA}

Luego de esta extensa revisión es prudente mencionar tres dificultades del uso de SMA y MBA en investigación. Primera, si el lector piensa que es un campo prometedor, tendrá que tener o adquirir el nivel relativamente alto de habilidades de programación que se requiere, bien sea para que programe directamente o trabaje en equipo. La segunda, la utilidad de la MBA para hacer grandes contribuciones a las ciencias sociales, solo es un problema si el lector no está convencido 
y es suspicaz. E1 campo no está aún bien delimitado, aunque este trabajo revisa en forma incompleta algunos caminos que ha seguido la investigación. La tercera es la diversidad de maneras de resolver un mismo modelo: algoritmos, programas, simulaciones. La falta de consenso sobre cómo y con qué resolver cada modelo es parte de este problema. A continuación comentamos cada uno de ellas.

Un recién graduado en economía debe aprender los fundamentos de programación, porque los programas académicos no suelen incluir este tipo de cursos. En un comienzo, los sofisticados modelos basados en agentes requerían saber programar en un lenguaje orientado a objetos, como Java o C++. Es decir, un nivel de habilidades superior al de la programación en hojas de cálculo. Desde entonces se han desarrollado algunos entornos de programación en que facilitan la tarea del científico social en la construcción de modelos, como Netlogo (Wilensky, 1999; ver también Larrosa, 2012). Pero este entorno de programación tiene limitaciones de escalabilidad ${ }^{11}$, aunque es una buena herramienta para crear prototipos y modelos de pequeña escala ${ }^{12}$.

La segunda dificultad es la actitud crítica de los economistas, y aun de investigadores en informática. ¿Es equiparable la validación de la ciencia social a la verificación de un programa? En informática, la validación y la verificación son diferentes, pero los científicos sociales suelen usarlos como sinónimos. Una crítica interesante contra el uso de MBA en ciencias sociales se encuentra en Nuno et al. (2005). Fagiolo et al. (2007) critican la validación empírica de los modelos MBA mediante la manipulación de hechos estilizados (ver Heine et al., 2005). Leombruni y Richiardi (2005) revisan los artículos sobre MBA en las principales revistas de economía y explican por qué los economistas académicos son reacios a usarlos. Las críticas y la indiferencia son un costo para el recién llegado que quiere dedicar su escaso tiempo a la modelación basada en agentes.

Un tercer problema, al que se ha prestado atención últimamente, es que la gran libertad que brinda la modelación basada en agentes da lugar a numerosos enfoques, lo que dificulta replicar todas las etapas de diseño: la elaboración de modelos que representen a los agentes, el idioma para ejecutar las simulaciones y, por supuesto, los resultados. En ausencia de criterios generales comprobados para determinar los

${ }^{11}$ Escalabilidad es la propiedad en virtud de la cual una simulación no pierde calidad a medida que aumenta el tamaño, por ejemplo, la cantidad de agentes que se incluyen.

${ }^{12}$ Railsback et al. (2006) evalúan otras opciones, y hacen una revisión más técnica de este y otros paquetes disponibles. Weidmann y Girardin (2005) analizan un conjunto menor de opciones para modelar MBA, con pruebas más técnicas del funcionamiento del software. 
métodos y protocolos, y sin un lenguaje de simulación compartido, esa libertad es la mayor limitación de los MBA para repetir y comparar los resultados de las investigaciones ${ }^{13}$. No obstante, en los últimos años algunos grupos de académicos han hecho grandes esfuerzo de coordinación, principalmente en biología (Grimm et al., 2006). Y han propuesto un protocolo llamado ODD (Overview, design concepts, and details) para orientar las publicaciones sobre MBA y los modelos respectivos, para facilitar la replicación de los experimentos y para mejorar la descripción de cómo opera cada modelo, respondiendo algunas preguntas básicas sobre el propósito (“¿qué hace este modelo?”), las entidades, las variables de estado y las escalas (“¿qué utiliza para hacerlo?”), la revisión y el cronograma (“¿qué hace qué y en qué orden?"), los criterios de diseño (“¿cuáles son los valores iniciales del modelo?"), los datos de entrada (“¿qué datos se deben ingresar?”) y los submodelos. Estos conceptos, con un vocabulario adaptado, ya se empiezan a utilizar en los modelos SMA y MBA aplicados a la economía (Wolf et al., 2013) y el mecadeo (Rand y Rust, 2011).

\section{CONCLUSIONES}

Los modelos de agentes computacionales permiten modelar directamente los problemas que enfrentan agentes con racionalidad limitada en ambientes delimitados. Muchos problemas económicos surgen en entornos de este tipo. Por ello, la MBA y los SMA son herramientas con gran potencial para simular fenómenos económicos relevantes, en los que la interacción repetida genera resultados de creciente complejidad.

Entre las ventajas de este método cabe mencionar las siguientes: 1) es adecuado para abordar el análisis de conceptos y realidades contemporáneos, como la heterogeneidad de los agentes económicos, la emergencia de comportamientos complejos y en apariencia imprevisibles, la no linealidad de las interrelaciones, la racionalidad limitada y las consecuencias inesperadas, en forma más intuitiva que los modelos formales tradicionales. Las crisis financieras recientes y las insuficiencias de los modelos de predicción muestran la necesidad de considerar la complejidad en el análisis económico (ver Stiglitz y Gallegatti, 2011);2) los agentes computacionales operan en ambientes imperfectos que se pueden representar mediante MBA;3) puesto que la MBA integra los niveles micro y macro de los procesos sociales,

${ }^{13}$ Windrum et al. (2007) analizan este problema y Heath et al. (2009), que revisan las publicaciones sobre SMA/MBA entre 1998 y 2008, encuentran diversas maneras de representar un modelo usando programas intérpretes o lenguajes de programación. 
puede fortalecer el diálogo entre disciplinas; 4) permite evaluar propuestas de política y sus efectos antes de que sean adoptadas; 5) ayuda a desarrollar, formalizar y evaluar teorías, arrojando luz sobre las alternativas teóricas que intentan explicar un mismo fenómeno; 6) hace posible realizar experimentos virtuales que no se pueden llevar a cabo en el mundo real.

En cuanto a las dificultades de la modelación basada en agentes es necesario mencionar que aún subsisten problemas de verificación y validación de los modelos. Los protocolos de programación tienden a la estandarización aunque los programas existentes generan o pueden generar resultados diferentes (verificación). Y aunque generaran resultados idénticos, los modelos pueden representar fenómenos reales de los que se carece de datos suficientes para compararlos con los resultados de la simulación (validación). No se puede dejar de mencionar que las jóvenes cohortes de científicos sociales tiene una gran dificultad por vacíos en su formación: la de aprender los lenguajes de programación y el manejo de los programas existentes. No obstante, este campo es una puerta abierta y un reto para la investigación interdisciplinaria.

\section{REFERENCIAS BIBLIOGFRÁFICAS}

1. Albino, V.; N. Carbonara e I. Giannoccaro. "Coordination mechanisms based on cooperation and competition within industrial districts: An agent-based computational approach", Journal of Artificial Societies and Social Simulation 6, 4, 2003, [http://jasss.soc.surrey.ac.uk/6/4/3.html].

2. Axelrod, R. The complexity of cooperation, Princeton, Princeton University Press, 1997.

3. Axelrod, R. y L. Tesfatsion. "A guide to newcomers to agent-based modeling in the social sciences", L. Tesfatsion y K. L. Judd, eds., Handbook of computational economics 2, Amsterdam, North-Holland, 2005.

4. Axtell, R., "Why agents? On the varied motivations for agent computing in the social sciences", working paper No. 17, Center on Social and Economic Dynamics, Washington, 2000.

5. Baptista, M. L.; C. R. Martinho et al. "An agent-based model of consumer behavior based on the BDI architecture and neoclassical theory", Developments in Business Simulation and Experiential Learning 41, 2014, pp. 170-178.

6. Boero, R.; M. Castellani y F. Squazzoni. "Labour market, entrepreneurship and human capital in industrial districts. An agent-based prototype", R. Leombruni y M. Richiardi, eds., Industry and labor dynamics. The agent-based computational economics approach, Singapur, World Scientific, 2004, pp. 332-349.

7. Bonabeau, E. "Agent-based modeling: Methods and techniques for simulating human systems", A. M. Sackler, ed., Colloquium of the National Academy of Sciences 99 3, 2002, pp. 7280-7287. 
8. Borrelli, F.; C. Ponsiglione et al. "Inter-organizational learning and collective memory in small firms clusters: An agent-based approach", Journal of Artificial Societies and Social Simulation 8, 3, 2005, [http:// jasss.soc.surrey.ac.uk/8/3/4.html].

9. Borrill, P. L. y L. Tesfatsion, "Agent-based modeling: The right mathematics for the social sciences?”, J. B. Davis y D. W. Hands, eds., Elgar companion to recent economic methodology, Cheltelham, Edward Elgar, 2011.

10. Brannon, E. L.; P. V. Ulrich et al. "Agent-based simulation of the consumer's apparel purchase decision”, National Textile Center Annual Report, noviembre de 2000, [http://www.ntcresearch.org/pdf-rpts/ anrp01/i98-a09-a1.pdf].

11. Brenner, T. "Simulating the evolution of localised industrial clusters - An identification of the basic mechanisms", Journal of Artificial Societies and Social Simulations 4, 3, 2001, [http://jasss.soc.surrey. ac.uk/4/3/4.html].

12. Bruun, C. "Agent-based Keynesian economics", R. Conte et al., eds., Simulating social phenomena, Berlín y Heidelberg, Springer, 1997.

13. Bruun, C. "Growth and inequality in agent-based models - Effects of introducing a wealth tax", presentado en la WEIHA Conference, Maastricht, junio de 2001.

14. Bruun, C. y F. Luna. "Endogenous growth with cycles in a Swarm economy: Fighting time, space, and complexity", F. Luna y B. Stefansson, eds., Economic simulations in Swarm. Agent-based modelling and object oriented programming, Amsterdam, Kluwer Academic Press, 2000.

15. Caramuta, L. R. y J. M. C. Larrosa. "Un enfoque de simulación basada en agentes para el estudio de senderos de compra dentro de un supermercado”, working paper, Universidad Nacional del Sur, 2007.

16. Chang, M.-H. y J. E. Harrington. "Agent-based models of organizations", L. Tesfatsion y K. L. Judd, eds., Handbook of computational economics 2, Amsterdam, North-Holland, 2005.

17. Collings, D.; A. Reeder et al. "Agent based customer modeling", Computing in economics and finance 1352, 1999.

18. Gatti, D.; E. Gaffeo et al. Emergent macroeconomics: An agent-based approach to business fluctuations, Milán, Springer, 2008.

19. Delre, S. A. y D. Parisi. "Information and cooperation in a simulated labor market: A computational model for the evolution of workers and firms", M. Salzano y D. Colander, eds., Complexity bints for economic policy, Milán, Springer, 2007, pp. 211-230.

20. Drogoul, A.; D. Vanbergue y T. Meurisse. "Multi-agent based simulation: Where are the agents?”, J. S. Sichman et al., eds., Multiagent-based simulation II, Berlín, Springer, 2003.

21. Edmonds, B. "The use of models - Making MABS actually work", S. Moss y P. Davidsson, eds., Multi-agent based simulation. Lecture notes in artificial intelligence [1979], Berlín, Springer, 2001.

22. Epstein, J. M. y R. Axtell. Growing artificial societies. Social science from the bottom-up, Cambridge, Mass., MIT Press, 1996. 
23. Fagiolo, G.; A. Moneta y P. Windrum. "A critical guide to empirical validation of agent-based models in economics: Methodologies, procedures, and open problems", Computational Economics 30, 2007, pp. 195-226.

24. Fagiolo, G.; G. Dosi y R. Gabriele. "Towards an evolutionary interpretation of aggregate labor market regularities", working paper, Laboratorio Revelli, Turín, 2004.

25. Farmer, J. D. y D. Foley. "The economy needs agent-based modelling”, Nature 460, 2009, pp. 685-686.

26. Fioretti, G. "Agent-based models of industrial clusters and districts", A. Tavidze, ed., Progress in economics research, vol. 9, Londres, Majestic Books, 2004, pp. 125-142.

27. Gabriele, R. "Labor market dynamics and institutions: An evolutionary approach", LEM working paper 7, Sant'Anna School of Advanced Studies, Pisa, 2002.

28. Galán, J. M.; L. R. Izquierdo et al. "Errors and artifacts in agentbased modelling", Journal of Artificial Societies and Social Simulation 12, 1, 2009, [http://jasss.soc.surrey.ac.uk/12/1/1.html].

29. Gallego, J. A. "Aplicaciones de la economía computacional y la teoría de la complejidad", Revista de Economia Institucional 9, 17, 2007, pp. 369-374.

30. Gatti, D.; E. Gaffeo et al. Emergent macroeconomics: An agent-based approach to business fluctuations, Milán, Springer, 2008.

31. Gilbert, N. Agent-based models, Thousan Oaks, Ca., Sage, 2008.

32. Gilbert, N. y K. G. Troitzsch. Simulation for the social scientist, Berkshire, Open University Press, 2005.

33. Gode, D. K. y S. Sunder. "Allocative efficiency of markets with zero-intelligence traders: Market as a partial substitute for individual rationality", Journal of Political Economy 101, 1, 1993, pp. 119-137.

34. González B., S. "The role of dynamics networks in social capital: A simulation experiment", tesis de maestría, Oxford University, 2004.

35. Grimm V.; U. Berger et al. "A standard protocol for describing individual-based and agent-based models", Ecological Modelling 198, 1-2, 2006, pp. 115-126.

36. Guimerà, R.; B. Uzzi et al. "Team assembly mechanisms determine collaboration network structure and team performance", Science 308, 5722, 2005, pp. 697-702.

37. Gulyás, L. Understanding emergent social phenomena. Methods, tools, and applications for agent-based modeling, tesis doctoral, Computer and Automation Research Institute, Budapest, 2005.

38. Heath, B.; R. Hill y F. Ciarallo. "A survey of agent-based modeling practices (January 1998 to July 2008)”, Journal of Artificial Societies and Social Simulation 12, 4, 9, 2009, [http://jasss.soc.surrey.ac.uk/12/4/9. html].

39. Heine, B.-O.; M. Meyer y O. Strangfeld. "Stylised facts and the contribution of simulation to the economic analysis of budgeting", Journal of Artificial Societies and Social Simulation 8, 4, 2005, [http:// jasss.soc.surrey.ac.uk/8/4/4.html]. 
40. Helbing, D. “Agent-based modeling”, D. Helbing, ed., Social selforganization, Berlín, Springer, 2012.

41. Heppenstall, A. J.; A. J. Evans et al. "The use of hybrid agent based systems to model petrol markets", Journal of Artificial Societies and Social Simulation 9, 3, 2006, [http://jasss.soc.surrey.ac.uk/9/3/2.html].

42. Heymann, D.; R. Perazzo y M. Zimmermann. Economías de fronteras abiertas: exploraciones en sistemas complejos, Buenos Aires, Teseo, 2013.

43. Huhns, M. y M. P. Singh. Readings in agents, San Mateo, Ca., Morgan Kaufmann, 1998.

44. Izquierdo, S.; L. Izquierdo et al. "Market failure caused by quality uncertainty", P. Mathieu et al., eds., Artificial economics, 2006, Berlín y Heidelberg, Springer, pp. 203-213.

45. Izquierdo, L.; J. M. Galán et al. "Modelado de sistemas complejos mediante simulación basada en agentes y mediante dinámica de sistemas", Empiria 16, 2008, pp. 85-112.

46. Jager, W. Modelling consumer behaviour, tesis doctoral, Centre for Environmental and Traffic Psychology, Rijksuniversiteit, Groninga, 2000.

47. Joo, K. H.; T. Kinoshita y N. Shiratori. "Design and implementation of an agent-based grocery shopping system", IEICE Transactions on Information and Systems E83, 11, 2000, pp. 1940-1951.

48. Kirman, A. P. "Whom or what does the representative individual represent?", Journal of Economic Perspectives 6, 2, 1992, pp. 117-136.

49. Kleijnen, J. P. "Verification and validation of simulation models", European Journal of Operational Research 82, 1, 1995, pp. 145-162.

50. Kydland, F. E. y E. C. Prescott. "The computational experiment: An econometric tool", Journal of Economic Perspectives 10, 1, 1996, pp. 69-85.

51. Larrosa, J. M. C. Economía computacional basada en agentes con aplicaciones en NetLogo, Bahía Blanca, EdiUNS, 2012.

52. Leombruni, R. y M. Richiardi "Why are economists sceptical about agent-based simulations?”, Physica A 355, 2005, pp. 103-109.

53. Leombruni, R. y M. Richiardi. "LABORsim: An agent-based microsimulation of labour supply. An application to Italy", Computational Economics 27, 1, 2006, pp. 63-88.

54. Li, H.; J. Sun y L. Tesfatsion. "Testing institutional arrangements via agent-based modeling: A U.S. electricity market application”, H. Dawid y W. Semmler, eds., Computational methods in economic dynamics, Berlín y Heidelberg, Springer, 2010.

55. Lucas, R. "Econometric policy evaluation: A critique", K. Brunner y A. Meltzer, eds., The Phillips curve and labor markets, Nueva York, Elsevier, 1976, pp. 19-46.

56. Macal, C. M. "Effects of global information availability in networks of supply chain agents", C. Macal et al., eds., Proceedings of the Agent 2003: Conference on Challenges in Social Simulation, Chicago, I1l., 2003.

57. Macal, C. M. y M. J. North. "A tutorial on agent-based modeling and simulation”, M. E. Kuhl et al., eds., Proceedings of the 2005 Winter Simulation Conference, Orlando, 2005.

58. Moss, S.; B. Edmonds y S. Wallis. "Validation and verification of computational models with multiple cognitive agents", report num- 
ber 97-25, Centre for Policy Modelling, Manchester Metropolitan University, 1997.

59. Neugart, M. "Labor market policy evaluation with ACE”, Journal of Economic Behavior E Organization 67, 2, 2008, pp. 418-430.

60. Nuno, D.; J. S. Sichman y H. Coelho. "The logic of the method of agent-based simulation in the social sciences: Empirical and intentional adequacy of computer programs", Journal of Artificial Societies and Social Simulation 8, 4, 2005, [http://jasss.soc.surrey.ac.uk/8/4/2.html].

61. Oremland, M. y R. Laubenbacher. "Using difference equations to find optimal tax structures on the SugarScape", Journal of Economic Interaction and Coordination 9, 2, 2014, pp. 233-253.

62. Pavón, J.; A. López-P. y J. M. Galán. “Modelado basado en agentes para el estudio de sistemas complejos”, Novática 218, 2012, pp. 13-16.

63. Pegoretti, G. et al. "An agent-based model of innovation diffusion: network structure and coexistence under different information regimes", Journal of Economic Interaction and Coordination 7, 2, 2012, pp. 145-165.

64. Pingle, M. y L. Tesfatsion. "Evolution of worker-employer networks and behaviors under alternative non-employment benefits: An agentbased computational study", A. Nagurney, ed., Innovations in financial and economic networks, Nueva York, Edward Elgar, 2003.

65. Railsback, S. F.; S. L. Lytinen y S. K. Jackson. "Agent-based simulation platforms: Review and development recommendations", Simulation 82, 9, 2006, pp. 609-623.

66. Rand, W. y R. Rust "Agent-based modeling in marketing: Guidelines for rigor", International Journal for Research in Marketing 28, 3, 2011, pp. 181-193.

67. Richiardi, M. "The promises and perils of agent-based computational economics”, working paper, Laboratorio Revelli, Turín, 2004.

68. Robertson, D. "Agent-based modeling toolkits", Academy of Management Learning and Education 4, 4, 2005, pp. 525-527.

69. Robertson, D. "Using agent-based models to manage the complex", K. Richardson, ed., Managing the complex: Philosophy, theory, and application, Greenwich, Information Age, 2005, pp. 419-432.

70. Russell, S. y P. Norvig. Artificial life: A modern approach, Upper Saddle River, NJ, Prentice Hall, 2004.

71. Sargent, R. G. "Verification and validation of simulation models", Proceedings of the 2010 Winter Simulation Conference, Baltimore, 2010.

72. Schelling, T. S. Micromotives and macrobehavior, Nueva York, Norton, 1978.

73. Schenk, T. A.; G. Löffler y J. Rauh. "Agent-based simulation of consumer behavior in grocery shopping on a regional level”, Journal of Business Research 60, 2007, pp. 894-903.

74. Schwaiger, A. y B. Stahmer. "SimMarket: Multiagent-based customer simulation and decision support for category management”, M. Schillo et al., eds., Multiagent system technologies, First German Conference MATES, Erfurt, Alemania, 2003, pp. 74-84. 
75. Siebers, P.-O. y U. Aickelin. "A multi-agent simulation of retail management practices", Proceedings of the 2007 Summer Computer Simulation Conference, San Diego, Ca., 2007.

76. Squazzoni F. y R. Boero. "Economic performance, inter-firm relations and local institutional engineering in a computational prototype of industrial sistricts", Journal of Artificial Societies and Social Simulation 5, 1, 2002, [http://jasss.soc.surrey.ac.uk/5/1/1.html].

77. Sterman, J. "Testing behavioral simulation models by direct experiment", Management Science 33, 12, 1989, pp. 1572-1592.

78. Stiglitz, J. E. y M. Gallegati. "Heterogeneous interacting agent models for understanding monetary economies", Eastern Economic Journal 37, 2011, pp. 6-12.

79. Tesfatsion, L. "How economists can get alife", W. B. Arthur et al., eds., The economy as an evolving complex system II, vol. XXVII, Santa $\mathrm{Fe}$ Institute Studies in the Science of Complexity, Addison-Wesley, 1997, pp. 533-564.

80. Tesfatsion, L. "Structure, behavior, and market power in an evolutionary labor market with adaptive search", Journal of Economic Dynamics and Control 25, 2001, pp. 419-457.

81. Tesfatsion, L. "Hysteresis in an evolutionary labor market with adaptive search", S.-H. Chen, ed., Evolutionary computation in economics and finance, Nueva York, Physica, 2002.

82. Tesfatsion, L. "Agent-based computational economics: A constructive approach to economic theory", L. Tesfatsion y K. L. Judd, eds., Handbook of computational economics 2, Amsterdam, North-Holland, 2005.

83. Tesfatsion, L. y K. L. Judd, eds., Handbook of computational economics 2. Agent-based computational economics, Amsterdam, Elsevier, 2006.

84. Twomey, P. y R. Cadman. "Agent-based modeling of consumer behavior in the telecoms and media markets", Info 4, 1, 2002, pp. 56-63.

85. Venables, M. y U. Bilge. "Complex adaptive modelling at J Sainsbury: The SimStore supermarket supply chain experiment", The LSE Strategy Eं Complexity Seminar, 30 de marzo de 1998.

86. Vinkovic, D. y A. Kirman. "A physical analogue of the Schelling model”, PNAS 103, 51, 2006, pp. 19261-19265.

87. Vriend, N. J. “ACE models of endogenous interactions”, L. Tesfatsion y K. L. Judd, eds., Handbook of computational economics 2, Agent-based computational economics, Amsterdam, Elsevier, 2006.

88. Weidmann, N. B. y L. Girardin. “Technical note: Evaluating Java development kits for agent-based modeling", Journal of Artificial Societies and Social Simulation 8, 2, 2005, [http://jasss.soc.surrey. ac.uk/8/2/8.html].

89. Wilensky, U. NetLogo, Center for Connected Learning and Computer-Based Modeling, Northwestern University, 1999, [http://ccl. northwestern.edu/netlogo/].

90. Windrum, P.; G. Fagiolo y A. Moneta. "Empirical validation of agent-based models: Alternatives and prospects", Journal of Artificial Societies and Social Simulation 10, 2, 2007, [http://jasss.soc.surrey. ac.uk/10/2/8.html]. 
91. Wolf, S.; J.-P. Bouchaud et al. "Describing economic agent-based models - Dahlem ABM documentation guidelines", Complexity Economics 2, 1, 2013, pp. 63-74.

92. Wooldridge, M. An introduction to multiagent systems, Londres, Wiley \& Sons, 2009.

93. Zhang, T. y D. Zhang, "Agent-based simulation of consumer purchase decision-making and the decoy effect", Journal of Business Research 60, 2007, pp. 912-922. 